\title{
Using IEC 61499 Models for Automatic Network Configuration of Distributed Automation Systems
}

\author{
Ben Schneider, Sebastian Voss, Monika Wenger, Alois Zoitl \\ fortiss, An-Institut Technische Universität München, Guerickestr. 25, \\ 80805 München, Germany (e-mail: \\ schneider/voss/wenger/zoitl@fortiss.org).
}

Keywords: distributed industrial automation, IEC 61499, Time-Sensitive Networking, real-time, deterministic networking, schedule synthesis

\section{INTRODUCTION}

Industrie 4.0 and IIoT introduce new challenges for distributed automation systems regarding the network. These requirments include the distribtion of control logic, a high degree of interoperability, the need for dynamic reconfiguration and software reusability. All these requirements can be fulfilled by the IEC 61499 which is a modeling language for distributed control systems and can be seen as the successor of IEC 61131. This trend towards highly distributed control systems also causes new challenges for the network, as the fastly growing number of network devices rises the complexity of their configuration. Also, mixed-criticality systems become more and more important which require a convergent network (4) i.e., the transmission of traffic with different characteristics on the same network cable, like deterministic real-time traffic for motion control next to traffic for data acquisition into the cloud. These new requirements can be fulfilled by the new Time-Sensitive Networking (TSN) Ethernet standard which is currently standardized by the IEEE 802.1 Working Group. However, the configuration of TSN devices is time consuming and error-prone because each networking device has to be configured separately (1). The first step towards automatic configuration of asynchronous, real-time capable networks is the analysis of IEC 61499's models (6) and their support for network modeling (w.r.t. timing behavior, criticality and complexity) and the identification of missing information, that is needed to generate network configurations.

\section{MODELING IN IEC 61499}

Figure 1 shows the Application and System Model and the relation between both which is represented by the Distribution Model. The Application Model contains the Function Block Network (FBN) representing the control code. The execution of FBs is triggered by the event connections (black lines between FBS in Figure 1). Multiple connected FBs form an event chain which defines the execution order of the FBs.

The System Model contains information about the physical composition of the distributed control system. This includes devices, network segments and the interface to the controlled industrial process. A device is for example

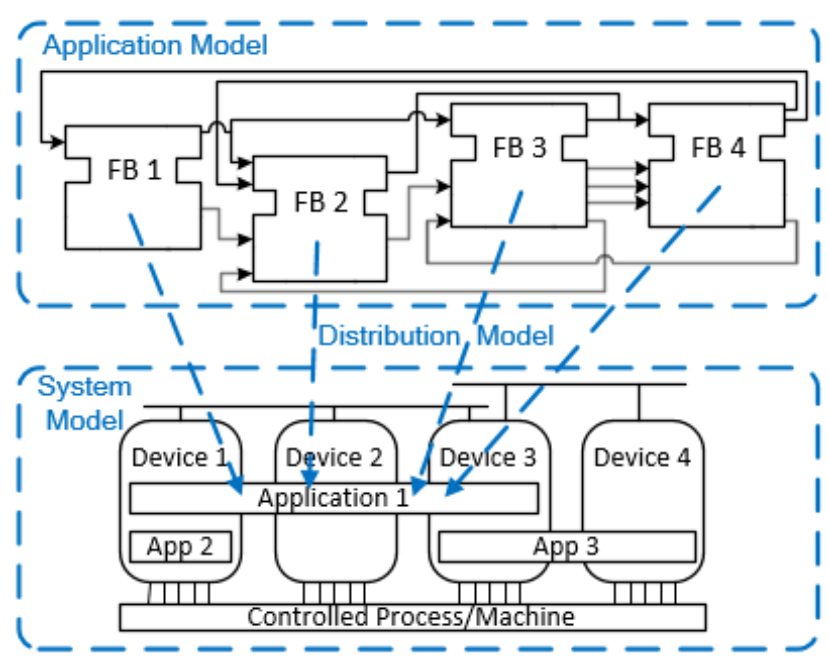

Fig. 1. Application, System and Distribution Model of IEC 61499

a specific Programmable Logic Controller (PLC) or an embedded computer and the network segment could specify the usage of Ethernet or a fieldbus like CAN or Profinet for communication.

After defining available devices and implementing the control algorithm as a Function Block Network (FBN), the application is mapped by the engineer to the control devices that are responsible for their execution. This mapping is the Distribution Model of IEC 61499 which is represented by the applications that are mapped to single or multiple devices like shown in Figure 1. During this process the network communication between the different devices and their application has to be modeled manually by the engineer using network Service Interface Function Blocks (SIFB) (3).

Each of these SIFBs has to be created separately, first by choosing the appropriate communication pattern such as publish-subscribe or client-server, second by choosing the protocol that shall be used for the communication e.g., UDP, MQTT or OPC UA and third by manually setting up the communication parameters such as IP address and port. After that the network devices need to be configured 
such that they meet the requirements of the distributed application.

\section{NETWORK MODELLING IN IEC 61499}

This configuration process is very time consuming and error-prone and can be automated by generating the necessary parameters for industrial communication directly from the models of IEC 61499. The first steps of modelling the network using concepts of IEC 61499 is shown in the next sections.

\subsection{Available Data for Network Modeling}

The Application Model provides the execution order of FBs which are defined by event chains. The type of the event source of a chain can also be derived to a cyclic source which periodically triggers the event chain or an asynchronous source without any periodic behavior. It is also possible to model deadline for real-time FBs (2). Furthermore, the communication pattern can be derived from the FBN where event connections between FBs refer to a publish-subscribe and adapters refer to a client-server pattern.

The System Model provides an overview of all available devices which are able to execute IEC 61499 compliant control software and the connection of different devices through network segments and corresponding links. Additional capabilities of entities (not only devices, also for segments, resources, etc.) can be modeled with Attributes described in IEC 61499 Annex G (6). Attributes are typically expressing vendor specific data like CPU type, supported communication protocols or runtime environment and more.

The Distribution Model provides the end-to-end connections of traffic streams and can be used to derive the appropriate parameters like IP address and port.

\subsection{Missing Data for Network Modeling}

The following paragraphs summarize requirements for missing data in the models of IEC 61499 needed to generate real-time capable network configurations.

The Application Model lacks a way to model the criticality of events between FBs. This mixed-criticality is necessary to model network determinism and can be used to derive network parameters like VLAN and priority for a stream in a TSN network. The sending times of events are optional information which can only be used in cyclic applications. The network transmission of events can be used to optimize the TSN schedule.

The current System Model does not give precise information of the network segments. The definition of a network segment in the IEC 61499-1 (6) is a "physical partition of a communication network". A higher accuracy of the description of network segments was not necessary when the standardization started, because most automation systems used a shared bus and where usually directly wired to a ring of devices. With new emerging networking technologies in the field of industrial automation such as TSN, a more precise segment needs to be defined. The only approach that seems suitable for a segment is to model it like a single networking device e.g., a switch, router or firewall. Segments and devices are connected via links which results in the network topology. Modeled data of a segment can for example be number of ports, link speed, bandwidth etc. The logical modeling of a segment, for example as a subnet containing one or more networking devices, is not applicable, because the standard already defines it as a physical partition.

The Distribution Model is not providing any possiblity to model the worst case execution time (WCET) of FBs which are mapped on different devices. This data is amongst others dependent on a more sophisticated device model.

\section{CONCLUSION AND FUTURE WORK}

This paper showed an analysis of network modeling capabilities in the Application, System and Distribution Model of IEC 61499. The goal is to provide an automatic configuration for networks including real-time schedules (e.g., for TSN). The next steps are to evaluate which already existing modeling languages in the automation domain (e.g., FDCML, EDDL, AML etc.) can be reused for network modelling in IEC 61499, implement a first prototype in $4 \mathrm{diac}^{1}$ and finally contribute to an extension of the IEC 61499 standard for network modeling based on the results of the prototype.

\section{ACKNOWLEDGEMENTS}

This work is partially funded by the German Federal Ministry of Education and Research (BMBF) under grant no. 01IS16022N through the project BaSys 4.0.

\section{REFERENCES}

[1] Marina Gutirrez, Astrit Ademaj, Wilfried Steiner, Radu Dobrin, Sasikumar Punnekkat, SelfConfiguration of IEEE 802.1 TSN Networks, IEEE Emerging Technologies and Factory Automation (ETFA), Limassol, 2017

[2] Alois Zoitl, Real-Time Execution for IEC 61499, ISA, 1st Edition, 2009

[3] L. Lednicki and J. Carlson A framework for generation of inter-node communication in componentbased distributed embedded systems, IEEE Emerging Technology and Factory Automation (ETFA), Barcelona, 2014, pp 1-8

[4] Sebastian Voss and Bernhard Schätz Deployment and Scheduling Synthesis for Mixed-Critical SharedMemory Applications, IEEE International Conference on Engineering of Computer based Systems (ECBS), Budapest, 2013, pp. 100-109

[5] R. Froschauer, F. Auinger, A. Schimmel and A. Zoitl, Engineering of communication links with AADL in IEC 61499 automation and control systems, International Conference on Industrial Informatics (INDIN), Cardiff, 2009, pp. 582-587

[6] IEEE IEC 61499, Function blocks - part 1: architecture, 2012, 2nd Edition

\footnotetext{
1 https://www.eclipse.org/4diac/
} 